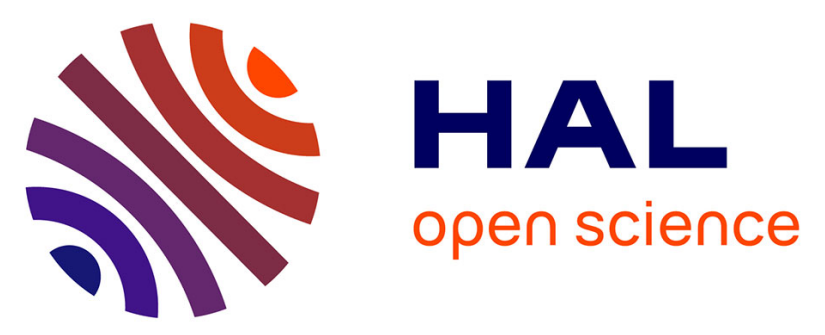

\title{
A hierarchical decision-making framework for the assessment of the prediction capability of prognostic methods
}

\author{
Zhiguo Zeng, Francesco Di Maio, Enrico Zio, Rui Kang
}

\section{To cite this version:}

Zhiguo Zeng, Francesco Di Maio, Enrico Zio, Rui Kang. A hierarchical decision-making framework for the assessment of the prediction capability of prognostic methods. Proceedings of the Institution of Mechanical Engineers, Part O: Journal of Risk and Reliability, 2017, 231 (1), pp.36 - 52. 10.1177/1748006X16683321 . hal-01632275

\section{HAL Id: hal-01632275 \\ https://hal.science/hal-01632275}

Submitted on 9 Nov 2017

HAL is a multi-disciplinary open access archive for the deposit and dissemination of scientific research documents, whether they are published or not. The documents may come from teaching and research institutions in France or abroad, or from public or private research centers.
L'archive ouverte pluridisciplinaire $\mathbf{H A L}$, est destinée au dépôt et à la diffusion de documents scientifiques de niveau recherche, publiés ou non, émanant des établissements d'enseignement et de recherche français ou étrangers, des laboratoires publics ou privés. 


\title{
A hierarchical decision making framework for the assessment of the prediction capability of
}

\author{
prognostic methods
}

Zhiguo Zeng a , Francesco Di Maio ${ }^{\mathrm{b}}$, Enrico Zio (*) ${ }^{\mathrm{a}, \mathrm{b}}$, Rui Kang ${ }^{\mathrm{c}}$

${ }^{a}$ Chair System Science and the Energy Challenge, Fondation Electricité de France (EDF), CentraleSupélec, Université Paris Saclay Chatenay-Malabry, 92290 France

${ }^{\mathrm{b}}$ Energy Department, Politecnico di Milano, Via La Masa 34, 20156, Milano, Italy

${ }^{\mathrm{c}}$ School of Reliability and Systems Engineering, Beihang University, Beijing, China

\section{Abstract}

In Prognostics and Health Management (PHM), the prediction capability of a prognostic method refers to its ability to provide trustable predictions of the Remaining Useful Life (RUL), with the quality characteristics required by the related maintenance decision making. The prediction capability heavily influences the decision maker's attitude towards taking the risk of using the predicted RUL to inform the maintenance decisions. In this paper, a four-layer, top-down, hierarchical decision making framework is proposed to assess the prediction capability of prognostic methods. In the framework, prediction capability is broken down into two criteria (Layer 2), six sub-criteria (Layer 3) and 19 basic sub-criteria (Layer 4). Based on the hierarchical framework, a bottom-up, quantitative approach is developed for the assessment of the prediction capability, using the information and data collected at the Layer-4 basic sub-criteria level. Analytical Hierarchical Process (AHP) is applied for the evaluation and aggregation of the sub-criteria and Support Vector Machine (SVM) is applied to develop a classification-based approach for prediction capability assessment. The framework and quantitative approach are applied on a simulated case study to assess the prediction capabilities of three prognostic methods of literature: fuzzy similarity, feed-forward neural network and hidden semi-Markov model. The results show the feasibility of the practical application of the framework and its quantitative assessment approach, and that the assessed prediction capability can be used to support the selection of the suitable prognostic method for a given application.

\section{Keywords}

Prognostic and Health Management (PHM), Remaining Useful Life (RUL), prediction capability, Analytical Hierarchical Process (AHP), fuzzy similarity, feed-forward neural network, hidden semi-Markov model 


\section{Introduction}

Prediction capability of a prognostic method refers to its ability to provide trustable predictions of the Remaining Useful Life (RUL), with the quality characteristics required by the related maintenance decision making. In Prognostics and Health Management (PHM), the predicted RUL (either by model-based prognostic methods [1-4] or data-driven prognostic methods [5-7]) is typically used by decision makers to schedule proper and timely maintenance. Usually, the decision makers choose between Condition-Based Maintenance $(\mathrm{CBM})$ policy $[8,9]$ or Preventive Maintenance $(\mathrm{PM})$ policy [10]. The choice of maintenance policies, then, depends on the decision makers' attitude to the risk of relying on a predicted RUL to plan maintenance services. Undoubtedly, such attitude is heavily influenced by the prediction capability of the prognostic method used. For instance, a prognostic method with high prediction capability might make the decision maker risk-prone, because he/she feels that he/she can trust the RUL predictions provided by the method. As a result, he/she is willing to take the risk of using them to plan PM. On the other hand, if the prediction capability of the prognostic method is not sufficient, the decision maker might be risk-averse towards using the RUL predictions to support any maintenance decision. Assessment of the prediction capability for a prognostic method is, then, an important task in PHM.

Performance Indicators (PPIs), based on test or benchmark data [11]. Most commonly used PPIs are related to the accuracy and precision of a prognostic method [12]. Accuracy PPIs quantify the closeness between the model prediction and the true measured values $[11,13]$. Precision PPIs measure how confident the model prediction is and the degree to which the prognostic method will yield the same results if repeatedly applied $[11,13,14]$. In general, good values of the PPIs give confidence to the decision makers about the predicted RUL, and make them prone to use the prediction results for supporting maintenance decisions. For example, by calculating some accuracy PPIs, Tobon-Micea et al. [15] compare the prognostic performance of a proposed wavelet-based prognostic method to that of a traditional time-domain method, and conclude that the new method can be applied to support CBM. Using accuracy PPIs, Micea et al. [16] compare the prognostic performances of two prognostic methods for application to Ni-MH-batteries. Hu et al. [17] develop an online assessment method for the PPIs of model-based prognostic methods. In [18], both accuracy and precision PPIs 
Diodes (LEDs). Other similar examples can be found for Lithium-Ion batteries [19], rotating machinery [20], composite laminates [21], etc., where accuracy and precision PPIs are used to compare the prognostic performances of different prognostic methods.

Although fundamental in practice, the existing PPIs reflect only one dimension of the prediction capability, i.e., the degree to which a prognostic method is able to explain the available data (referred to as the prediction performance in this paper) $[22,23]$. Indeed, the prediction capability of a prognostic method is also influenced by the trustworthiness of the method, which is defined in this paper as the confidence that the prognostic method can provide an accurate and precise RUL, with correct and fair quantification of its related uncertainty. Such confidence comes from our knowledge on the prognostic method, such as its proven records of successful applications on similar problems or our knowledge on its inherent methodological characteristics in relation to RUL predictions. Suppose that two prognostic methods, denoted by method A and method B respectively, perform equally well in terms of prediction performances (measured by the PPIs computed on the same available data); while method A has been applied successfully in various scenarios of setting similar to the one of interest, method B is newly developed and has rarely been applied before: it seems reasonable that in this situation, a decision maker would prefer to implement and use method A to support maintenance decisions. performance (in terms of PPIs) and the trustworthiness of the prognostic method should be considered. Whereas the assessment of the prognostic performance is relatively mature through the quantification of PPIs $[12,14,22]$, the assessment of the trustworthiness of the prognostic method deserves further consideration. In literature, the trustworthiness of a method or a process is often measured in terms of its maturity [24, 25]. The concept of maturity originated in the 1970s, when a model was developed to assess the maturity of an information system's function [26]. Later, the Software Engineering Institute (SEI) developed the Capability Maturity Model (CMM) to assess the maturity of a process for developing software with desirable quality/reliability/trustfulness characteristics [27]. Based on the CMM, a Prediction Capability Maturity Model (PCMM) has been recently developed to assess the maturity of modeling and simulation efforts [24]. Other approaches of maturity assessment are being developed and applied in different areas, e.g., master data maturity assessment [28], enterprise risk management [29], hospital information system [30], etc. However, 
there is no existing maturity assessment methods in the specific context and for the specific aim of prognostics and maintenance decision making.

To this aim, in this paper, we consider both the prediction performance and the method trustworthiness to assess the prediction capability of a prognostic method. It should be noted that an initial effort on prediction capability assessment was published by the authors in [31], however without considering the contribution of method trustworthiness and using only a simple weighted average of the PPIs to quantify prediction quality.

The rest of the paper is organized as follows. The hierarchical framework is presented in Section 2 and, then, used in Section 3 to assess the prediction capabilities of three data-driven prognostic methods of literature. In Section 4, we draw some conclusions and give some ideas of future research.

\section{Hierarchical framework and assessment approach}

We present the hierarchical framework developed to assess the prediction capability of prognostic methods in Subsection 2.1, considering two main attributes, RUL prediction quality and method trustworthiness. Prediction quality is assessed in Subsection 2.2 and AHP method is applied in Subsection 2.3 to assess the method trustworthiness. In Subsection 2.4, a classification-based method is developed to determine the prediction capability based on the prediction the quality and method trustworthiness.

\subsection{Framework of prediction capability assessment}

We present a four-layer hierarchical model to support the assessment of prediction capability, as shown in Figure 1. The prediction capability represented by $C$ (Layer 1 in Figure 1) is characterized in terms of RUL prediction quality and prognostic method trustworthiness (Layer 2 in Figure 1). The former, represented by $Y$, measures the performance of the prognostic method with respect to the specific application and data, while the latter, represented by $X$, measures the confidence based on knowledge related to the fact that the prognostic method provides trustworthy predictions, in terms of point estimates and uncertainty quantifications. The inhibit (conditional) gate indicates the logical relationship between $X$ and $Y$ in determining the prediction capability: to have a good prediction capability, the prognostic method should at least satisfy a minimum requirement of prediction quality; once this minimum requirement is satisfied, the prediction capability is determined jointly by the prediction quality and the method trustworthiness. 


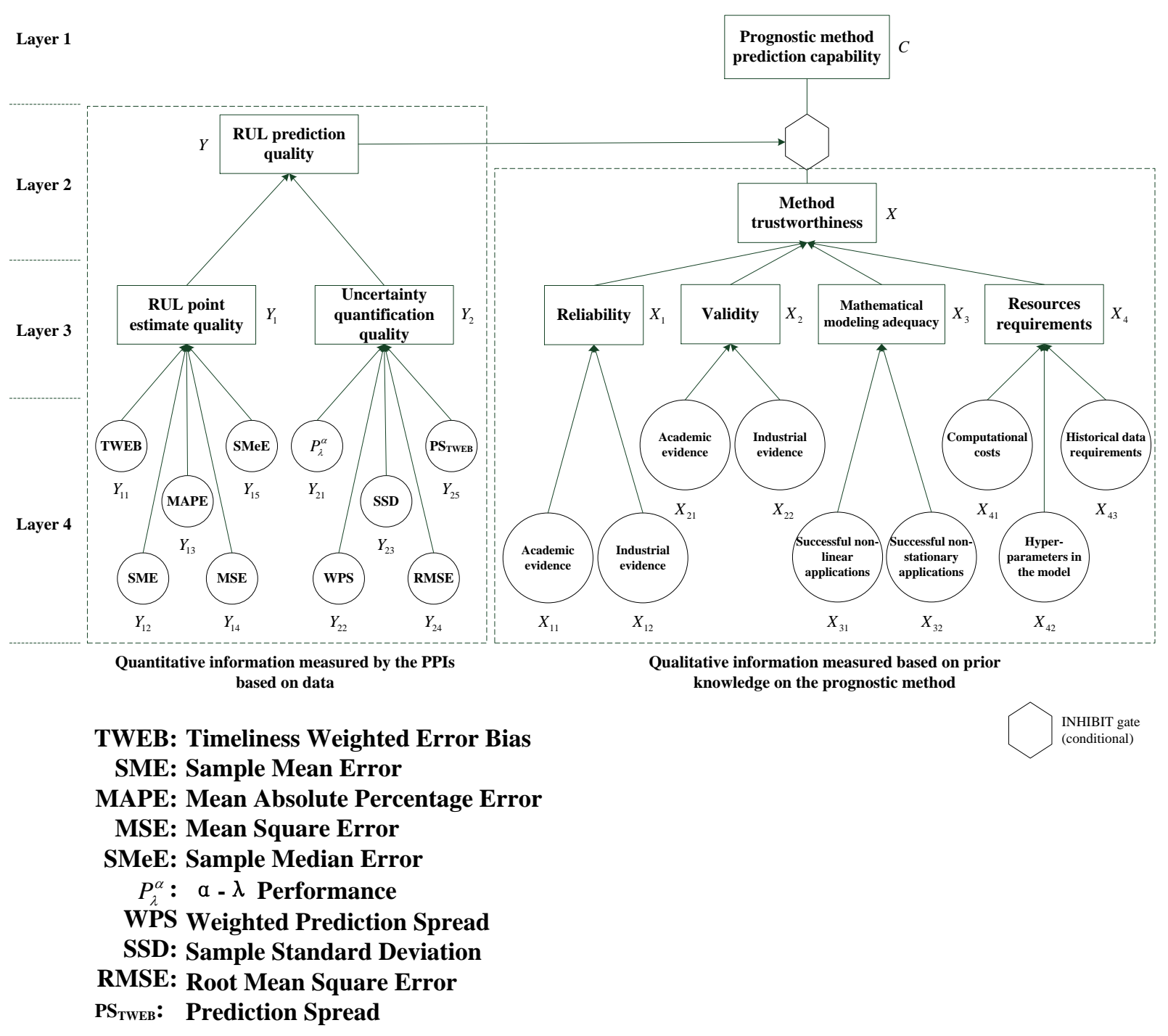

Figure 1 Hierarchical framework for prediction capability assessment

The two attributes in Layer 2 are further broken down into factors that influence them, leading to the six criteria in Layer 3: RUL point estimate quality $\left(Y_{1}\right)$ and uncertainty quantification quality $\left(Y_{2}\right)$, which contribute to the RUL prediction quality and reliability $\left(X_{1}\right)$, validity $\left(X_{2}\right)$, mathematical modeling adequacy $\left(X_{3}\right)$ and resources requirements $\left(X_{4}\right)$, which influence the method trustworthiness. Detailed descriptions of the criteria are given in Table 1. 


\begin{tabular}{|c|c|c|}
\hline Notation & Meaning & Description \\
\hline$Y_{1}$ & $\begin{array}{l}\text { RUL point } \\
\text { estimate quality }\end{array}$ & $\begin{array}{l}\text { Measures the distance of the RUL point estimates from the true RUL values, i.e., } \\
\text { the accuracy of the prognostic method. An accurate prognostic method is obviously } \\
\text { preferred. }\end{array}$ \\
\hline$Y_{2}$ & $\begin{array}{c}\text { Uncertainty } \\
\text { quantification } \\
\text { quality }\end{array}$ & $\begin{array}{l}\text { Measures the spread and variability of the RUL predictions, i.e., the precision of the } \\
\text { prognostic method. A precise prognostic method is preferred. }\end{array}$ \\
\hline$X_{1}$ & Reliability & $\begin{array}{l}\text { Measures the capability of the method to yield the same RUL prediction quality, } \\
\text { when different analysts apply it on similar sets of data related to similar problems: } \\
\text { the larger the reliability, the more trustworthy the method is. }\end{array}$ \\
\hline$X_{2}$ & Validity & $\begin{array}{l}\text { Measures the capability of the method to achieve the same RUL prediction quality, } \\
\text { when applied to solve different problems with similar characteristics: the larger the } \\
\text { validity, the more trustworthy the method is for use in different problems of similar } \\
\text { characteristics. }\end{array}$ \\
\hline$X_{3}$ & $\begin{array}{l}\text { Mathematical } \\
\text { modeling } \\
\text { adequacy }\end{array}$ & $\begin{array}{l}\text { Measures the capability of the method to deal with problems of given complexity: a } \\
\text { less advanced method may handle well linear and simplified problems, whereas a } \\
\text { more complex and advanced method is needed to deal with more realistic problems, } \\
\text { e.g., nonlinear and non-stationary problems. In these situations, such methods } \\
\text { would be more adequate and trustworthy compared to less mathematically complex } \\
\text { and advanced methods. }\end{array}$ \\
\hline$X_{4}$ & $\begin{array}{l}\text { Resources } \\
\text { requirements }\end{array}$ & $\begin{array}{l}\text { Measures the required resources by the prognostic methods, e.g, the data } \\
\text { requirements, the computational costs, the number of hyper-parameters, etc. A } \\
\text { prognostic method with lower resource requirements is more controllable and } \\
\text { verifiable during the training phase under the realistic available data, and therefore, } \\
\text { is more trustworthy for such settings. }\end{array}$ \\
\hline
\end{tabular}

The six criteria in Layer 3 are further decomposed into a layer of 19 basic sub-criteria (Layer 4 in Figure 1), where data and information can be used to support the assessment of prediction capability. Detailed descriptions of all the 19 basic sub-criteria can be found in the Appendix. Depending on the nature of the basic sub-criteria, they might take either numerical or linguistic values. The basic sub-criteria used to evaluate the RUL prediction quality are, in fact, quantitative PPIs related to accuracy and precision of a prognostic method. All of them take numerical values, e.g., the Timeless Weighted Error Bias (TWEB, $Y_{11}$ ) in Figure 1. The basic sub-criteria used to evaluate the method trustworthiness, on the other hand, represent evidence on various aspects of the trustworthiness of the prognostic method. Some of them are objective in nature, and, therefore, can be measured by numerical indicators, e.g., the number of academic evidence $\left(X_{11}\right)$ in Figure 1 . Others are qualitative in nature and can only be represented by linguistic or non-numerical values: evaluation of these basic sub-criteria requires the involvement of subjective judgements.

To assess the prediction capability, data and information are collected to support the evaluation of the Layer 4 basic sub-criteria ( $X_{i j}$ and $Y_{i j}$ ). Then, the basic sub-criteria are aggregated to assess the criteria in Level-3, and 
1 further aggregated to assess the Level-2 attributes of prediction quality and method trustworthiness. Finally, the prediction capability of the prognostic method is determined based on the joint contributions of the two Level-2 attributes, as shown in Figure 2. The obtained prediction capability incorporates the influences from both prediction quality and method trustworthiness, and, therefore, can be used to support the selection of appropriate prognostic methods for given maintenance planning requirements.

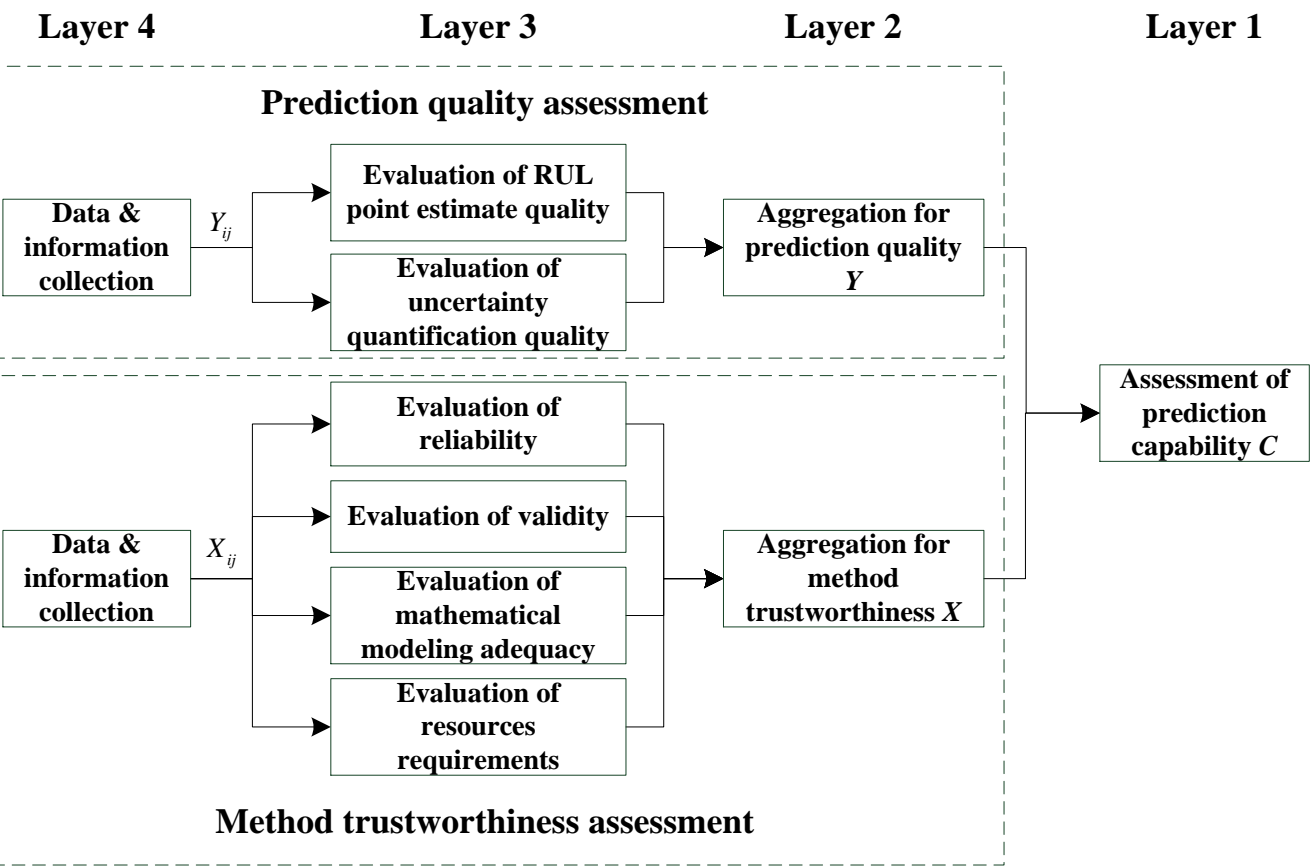

Figure 2 Procedures for prediction capability assessment

\subsection{Prediction quality assessment method}

As shown in Figure 2, the assessment of prediction quality starts from collecting data and information for the Level-4 basic sub-criteria related to prediction quality. Various numerical indicators, referred to as Prognostic Performance Indicators (PPIs), have been defined in literature to assess the performance of a RUL prediction method with respect to both point estimates and uncertainty quantifications. In this work, the PPIs listed in Table A.1 are adopted as the Layer-4 basic sub-criteria; their values, denoted by $Y_{i j}$, $i=1,2, j=1,2, \ldots, 5$, are calculated based on the formula listed in Table A.1.

Next, the PPIs are aggregated to evaluate the two Layer-3 criteria related to prediction quality, i.e., the RUL point estimate quality and uncertainty quantification quality. As shown in Table A.1, the values of all the $Y_{i j} \mathrm{~s}$ are bounded in the range $(-\infty, 1]$. A weighted-average method is used to aggregate the Layer-4 basic 
sub-criteria:

$$
Y_{i}=\sum_{j=1}^{n_{i}} \omega_{i j} \cdot Y_{i j}, i=1,2, j=1,2, \ldots, n_{i},
$$
where $n_{i}, i=1,2$ denotes the number of the Layer-4 basic sub-criteria associated with the $i$ th Layer-3 sub-criteria and $\omega_{i j}, j=1,2, \ldots, n_{i}$ are the weights of the Layer-4 basic sub-criteria. In this paper, we have $n_{1}=n_{2}=5$, and $\sum_{j=1}^{n_{i}} \omega_{i j}=1, i=1,2$. The weights represent the relative contribution of a basic sub-criteria to the corresponding Layer-3 criterion. In practice, the weights can be obtained by expert assessments or through some structured analysis method, e.g., the Analytical Hierarchical Processes (AHP) method [32]. It is easy to verify from (1) that both $Y_{1}$ and $Y_{2}$ take values in $(-\infty, 1]$, where a value close to 1 indicates good performance.

The two Layer-3 criteria are again aggregated to yield the prediction quality $Y$ by the weighted average:

$$
Y=\exp \left\{\sum_{i=1}^{2} \omega_{i} \cdot Y_{i}-1\right\}
$$

11 where $\omega_{i}$ is the weight for $Y_{i}$ and $\sum_{i=1}^{2} \omega_{i}=1$. As for the weights in the Layer-3 calculations, the $\omega_{i}$ can also be determined by experts assessments based on structured analysis methods such as the AHP method [32]. The exponential function in (2) is used for normalization: since $Y_{i} \in(-\infty, 1]$, it is easy to verify that $Y \in(0,1]$ and a value close to 1 indicates good prediction quality.

\subsection{Method trustworthiness assessment method}

Since the assessment of method trustworthiness involves multiple quantitative (i.e., the $X_{11}-X_{32}$ in Figure 1) and qualitative sub-criteria (i.e., the $X_{41}-X_{43}$ in Figure 1), it is formulated as a Multi-Criteria Decision Analysis (MCDA) problem [33]. As a widely applied MCDA method [34], AHP is selected for the assessment. AHP, first introduced in 1977 [32], is a hierarchical framework to support multi-criteria decision analysis, where the decision problem considered (the first, top, layer in the hierarchy) is decomposed into several layers of criteria and, eventually, the last, bottom layer containing the alternatives available for the solution of the decision problem. be ranked with respect to the decision problem in the top layer [32]. For a detailed discussion on the implementation procedures of AHP, readers might refer to $[35,36]$. The AHP model for method trustworthiness assessment is illustrated in Figure 3. 


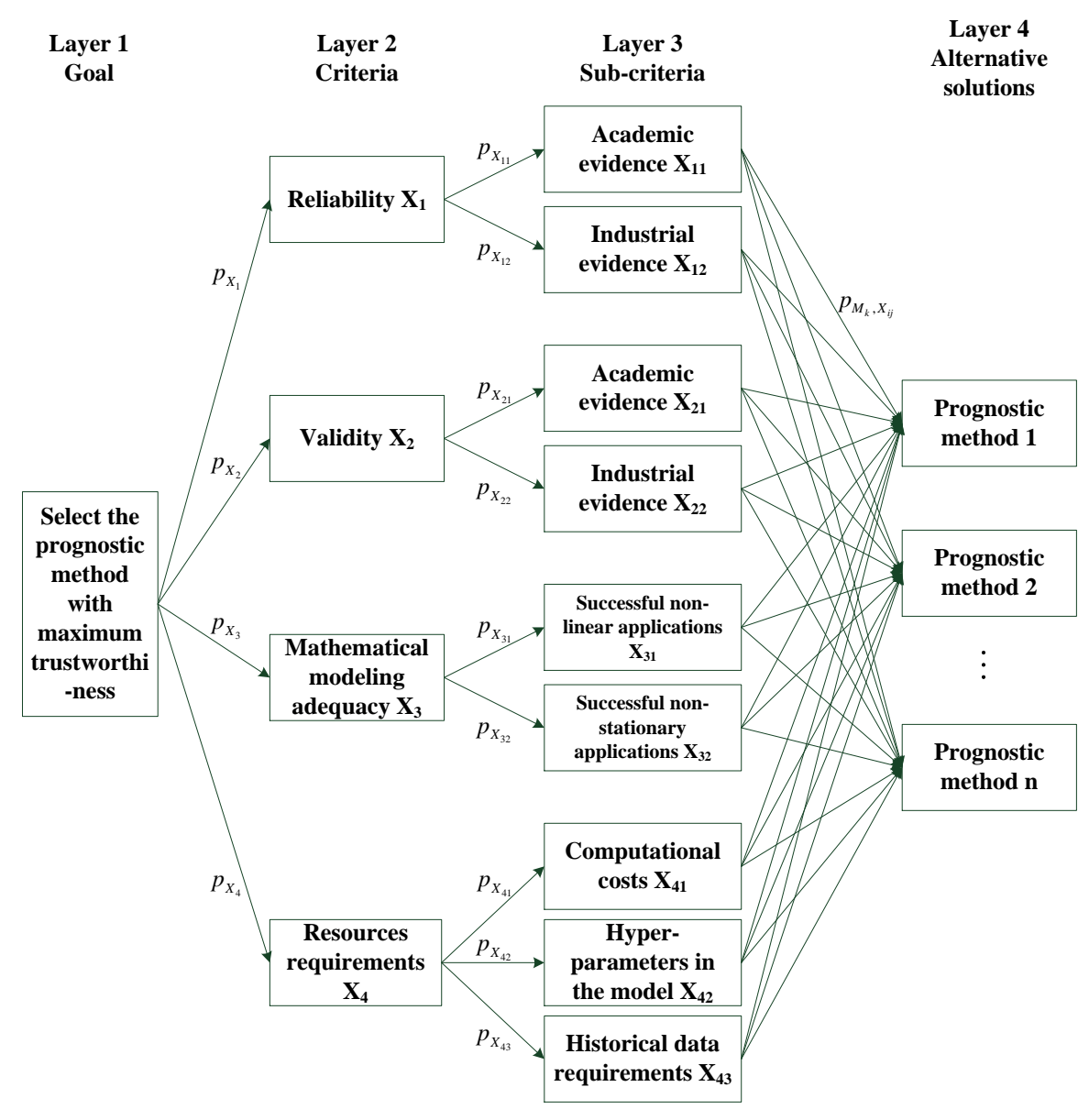

Figure 3 The AHP model for trustworthiness assessment

Based on the AHP model of Figure 3, the assessment of method trustworthiness involves three steps:

Step 1: Determine the inter-level priorities for the criteria $\left(p_{X_{i}}\right)$, sub-criteria $\left(p_{X_{i j}}\right)$ and alternative solutions

$5\left(p_{M_{k}, X_{i j}}\right)$. The inter-level priorities quantify the relative importance of the lower-level elements with respect to the

6 corresponding high-level element. For the qualitative sub-criteria, experts compare their relative importance using

7 the 1-9 scaling system defined in [37], where scale 9 represents " $i$ is extremely more important than $j$ ", scale

81 represents "equally important" and scale $1 / 9$ represents " $j$ is extremely more important than $i$ ". Pairwise

9 comparison matrices, indicated with the symbol $A$ in this paper, are constructed by filling out each element $a_{i j}$

10 with the numerical value of relative importance and considering the reciprocity property, which indicates that $a_{i j}=1 / a_{j i}$.

For the quantitative basic sub-criteria $X_{11}-X_{32}$, their inter-level priorities can be determined by calculating

priority weights as: 


$$
p_{M_{k}, X_{i j}}=\frac{X_{M_{k}, X_{i j}}}{\sum_{k=1}^{n} X_{M_{k}, X_{i j}}},
$$

where $p_{M_{k}, X_{i j}}$ is the inter-level priority of the $k$ th prognostic method with respect to the basic sub-criteria $X_{i j}$, and $X_{M_{k}, X_{i j}}$ is the numerical value that the $k$ th prognostic method takes with respect to the basic sub-criteria $4 \quad X_{i j}$.

Once the comparison matrix for a given level of the hierarchy has been constructed, the eigenvalue method is used to calculate the inter-level priorities [32]. Suppose the priorities associated with a comparison matrix $A$ are denoted by $\mathbf{p}=\left[p_{L, 1}, p_{L, 2}, \cdots, p_{L, n}\right]^{T}$. The eigenvalue method first calculates the eigenvector of $A$ that corresponds to the largest eigenvalue, denoted by $\mathbf{p}_{M}$ and $\lambda_{M}$, respectively. The priority vector $\mathbf{p}$ is, then,

9 calculated by normalizing the vector $\mathbf{p}_{M}$, as in (4) below, where $\mathbf{p}_{(i)}$ and $\mathbf{p}_{M,(i)}$ represent the $i$ th component in $\mathbf{p}$ and $\mathbf{p}_{M}$, respectively:

$$
\begin{aligned}
& \mathbf{p}_{(i)}=\frac{\mathbf{p}_{M,(i)}}{\mathbf{p}_{M}^{T} \cdot \mathbf{p}_{M}}, i=1,2, \ldots, n, \\
& A \mathbf{p}_{M}=\lambda_{M} \mathbf{p}_{M} .
\end{aligned}
$$

Finally, the consistency of the comparison matrix is checked to see if the calculated priority vector makes sense. A comparison matrix $A$ is consistent if it satisfies both the reciprocity rule [33]:

$$
a_{i j}=\frac{1}{a_{j i}}
$$

and the transitivity rule [33]:

$$
a_{i j}=a_{i k} \times a_{k j},
$$

17 where $a_{i j}$ is the element in the $i$ th row and $j$ th column of $A$ and $i, j, k$ are indexes for the criteria or 18 alternative solutions in $A$. The consistency can be checked following the procedure in Figure 4, where $R I$ is the $19 C I$ (Confidence Index) of a randomly generated $n \times n$ matrix whose values can be found in [32, 37]. The 20 three-step procedures are repeated for each criteria, sub-criteria and alternative solutions, until all the $p_{X_{i}}, p_{X_{i j}}$ 21 and $p_{M_{k}, X_{i j}}$ are determined. 


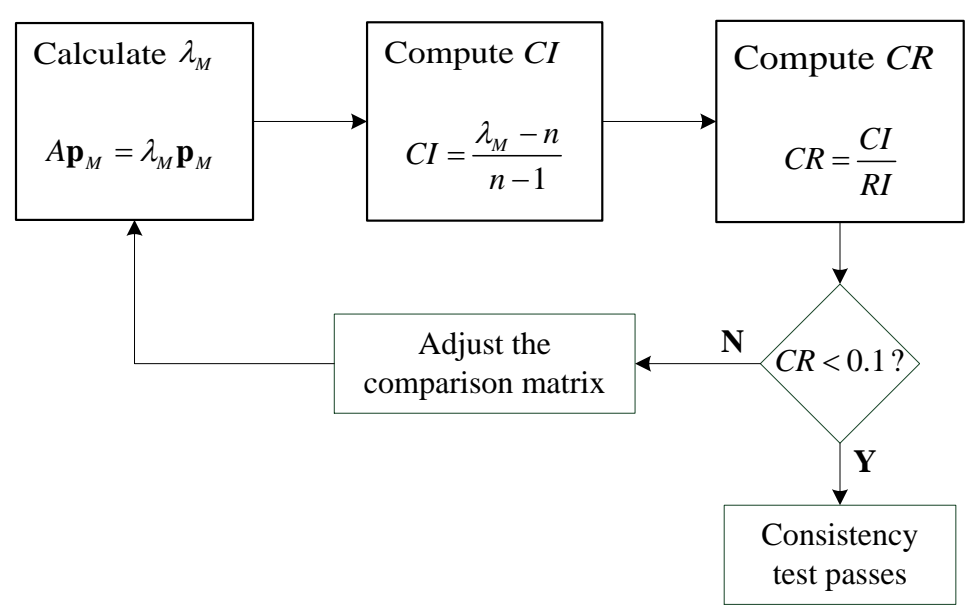

Figure 4 Procedures for consistency tests [32, 37]

Step 2: Calculate the global priority for each alternative solution.

A bottom-up synthesis process is used to calculate the global priority for each alternative solution, with respect to the top goal of the hierarchy:

$$
p_{M_{k}}=\sum_{i=1}^{4} \sum_{j=1}^{n_{X_{i}}} p_{X_{i}} \cdot p_{X_{i j}} \cdot p_{M_{k}, X_{i j}},
$$

where $p_{M_{k}}$ is the global priority of the $k$ th prognostic method and $n_{X_{i}}$ is the number of sub-criteria under the criterion $X_{i}$. Note that the global priorities should sum up to 1 , i.e., $\sum_{i=1}^{n} p_{M_{i}}=1$.

Step 3: Determine the method trustworthiness.

The method trustworthiness for each prognostic method, denoted by $X_{M_{i}}, i=1,2, \cdots, n$, is then determined based on the global priorities:

$$
X_{M_{i}}=\frac{p_{M_{i}} \cdot X_{\max }}{\max _{i=1}^{n}\left(p_{M_{i}}\right)},
$$

where $X_{\max }$ is the method trustworthiness of the prognostic method with the largest global priority, which is evaluated based on expert judgements. The value of $X_{\max }$ ranges in $[0,1]$, where a value closer to 1 indicates that the prognostic method is more trustworthy.

\subsection{Prediction capability assessment and prognostic method selection}

Prediction capability $C$ is an integrated metric that supports the selection of appropriate prognostic methods for a given application scenario. Depending on the role that the predicted RUL plays in maintenance 
planning, three typical application scenarios are usually distinguished: fully supportive, where the predicted

2 RUL is used to support Predictive Maintenance (PM) planning; partially supportive, where the predicted RUL

3 is used to support Condition-Based Maintenance (CBM) planning; and non-supportive, where the predicted

4 RUL is not directly applicable in maintenance planning. Therefore, the prediction capability is assumed to

5 take three discrete values, $C \in\left\{C_{0}, C_{1}, C_{2}\right\}$, where $C_{0}, C_{1}, C_{2}$ correspond to the required prediction

6 capability for the non-supportive, partially supportive and fully supportive application scenarios, respectively.

7 The issue of prediction capability assessment, is, then, formulated within a classification framework: given a prognostic method, which is characterized in terms of prediction quality and method trustworthiness, select among the above three candidates a proper value for its prediction capability.

In this paper, we assume that training data are available to construct a classifier for prediction capability assessment using supervised learning algorithms. The training data comprise of prognostic methods with known prediction quality, method trustworthiness and prediction capability. In Figure 5, we present 200 training data, which are constructed by randomly generating 200 samples of $X$ and $Y$, and then, inviting

14 decision makers to assess the prediction capability for each combination of $X$ and $Y$. Support Vector Machine (SVM) is used to construct a classifier for prediction capability assessment. We directly apply the SVM algorithm in MATLAB ${ }^{\circledR}$ R2015b and the result is shown in Figure 6. A 10-fold cross validation is conducted to validate the classifier. The average misclassification rate of the classifier is $\epsilon_{1}=0.04$, which indicates good classification performance. The $X-Y$ plane is partitioned in non-supportive, partially supportive and fully supportive regions, corresponding to $C=C_{0}, C=C_{1}$ and $C=C_{2}$, respectively. The prediction capability of a prognostic method can, then, be determined based on its position in the $X-Y$ plane of Figure 6. 


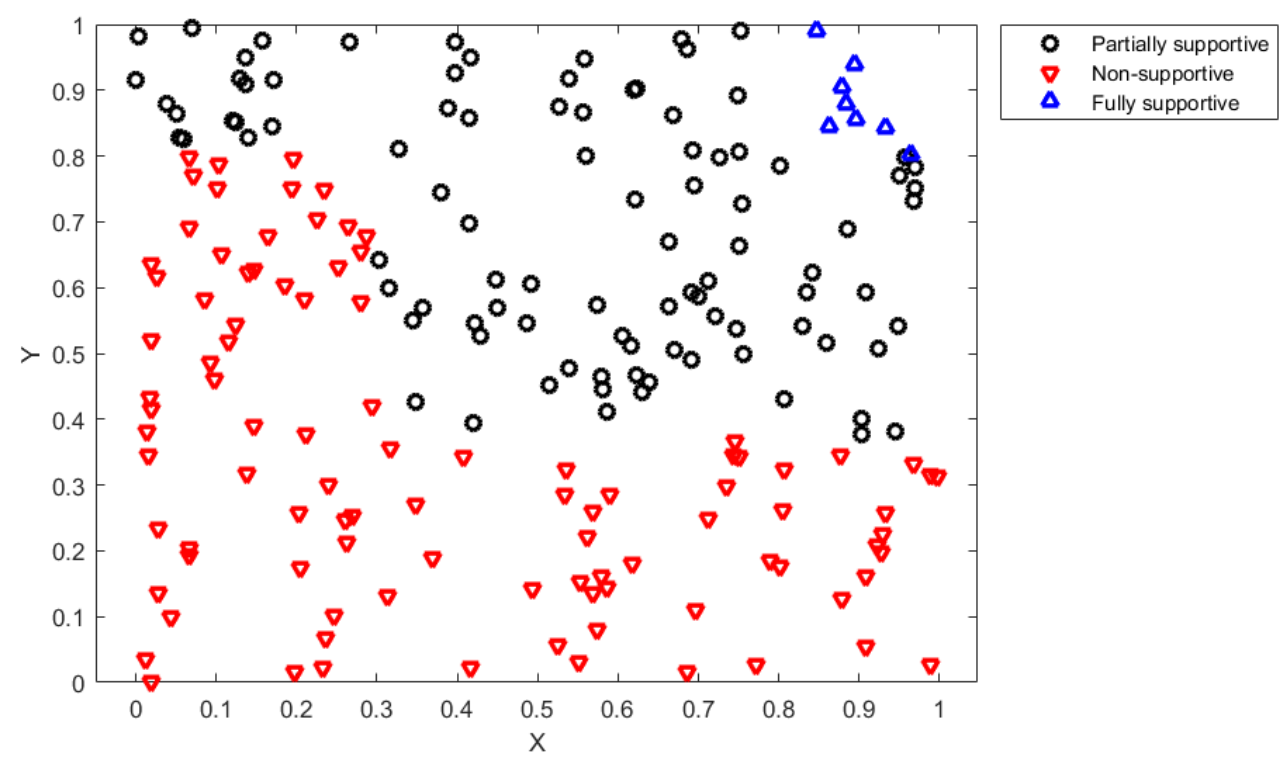

\section{Figure 5 Training data for prediction capability assessment}

Figure 6 reflects, based on the training data, the decision makers attitude to the risk of relying on a predicted RUL to plan maintenance services. It can be seen from Figure 6 that to be qualified to support PM, the decision maker thinks that a prognostic method needs to have both high prediction quality and high trustworthiness (fully supportive region). Also, when $Y \leq e^{-1}$ (roughly speaking, it means that the average prediction error between the predicted and true RUL is higher than the total life, see Table A.1 and Eq. (2)), the decision maker is not willing to apply the prognostic method to support any kind of maintenance decisions (non-supportive region), regardless of how well the method trustworthiness is. This fact is also reflected by the conditional gate in Figure 1. If the minimum requirement of $Y$ is satisfied $\left(Y>e^{-1}\right)$, the prediction capability further depends on the value of method trustworthiness: if the method trustworthiness is medium or high (roughly speaking, $X \geq 0.3$ ), the decision maker would apply the method to support CBM (partially supportive region); otherwise, only with higher prediction quality (roughly speaking, $Y>0.8$ ), the prognostic method can be qualified to support CBM. 


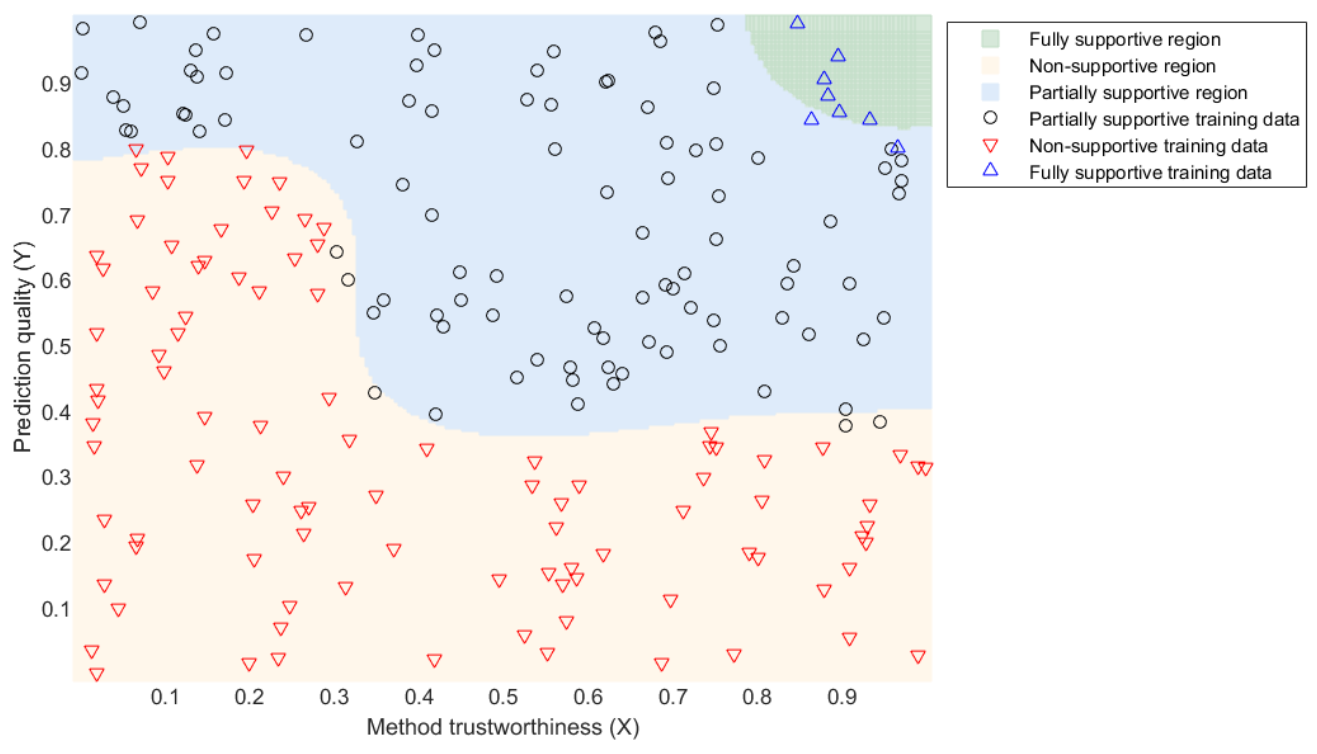

Figure 6 A classifier constructed for prediction capability assessment

3 A major strength of the developed prediction capability assessment framework is that it integrates both

4 prediction quality and method trustworthiness of the prognostic method, while existing frameworks, such as 5 those in [12] or [14], often neglect method trustworthiness. To demonstrate the strength of the developed 6 framework, we also apply the prediction quality based framework on the training data in Figure 5. Since only 7 the prediction quality is considered, we only use $Y$ to construct the classifier. We again use SVM to 8 construct the classifier and the result is given Figure 7. A 10-fold cross validation is conducted. The average 9 misclassification rate for this classifier is $\epsilon_{2}=0.22$, which is much larger than that of the developed 10 framework $\left(\epsilon_{1}=0.04\right)$. The comparison shows that by considering the method trustworthiness, the developed 11 assessment framework provides a more comprehensive description of the prediction capability. 


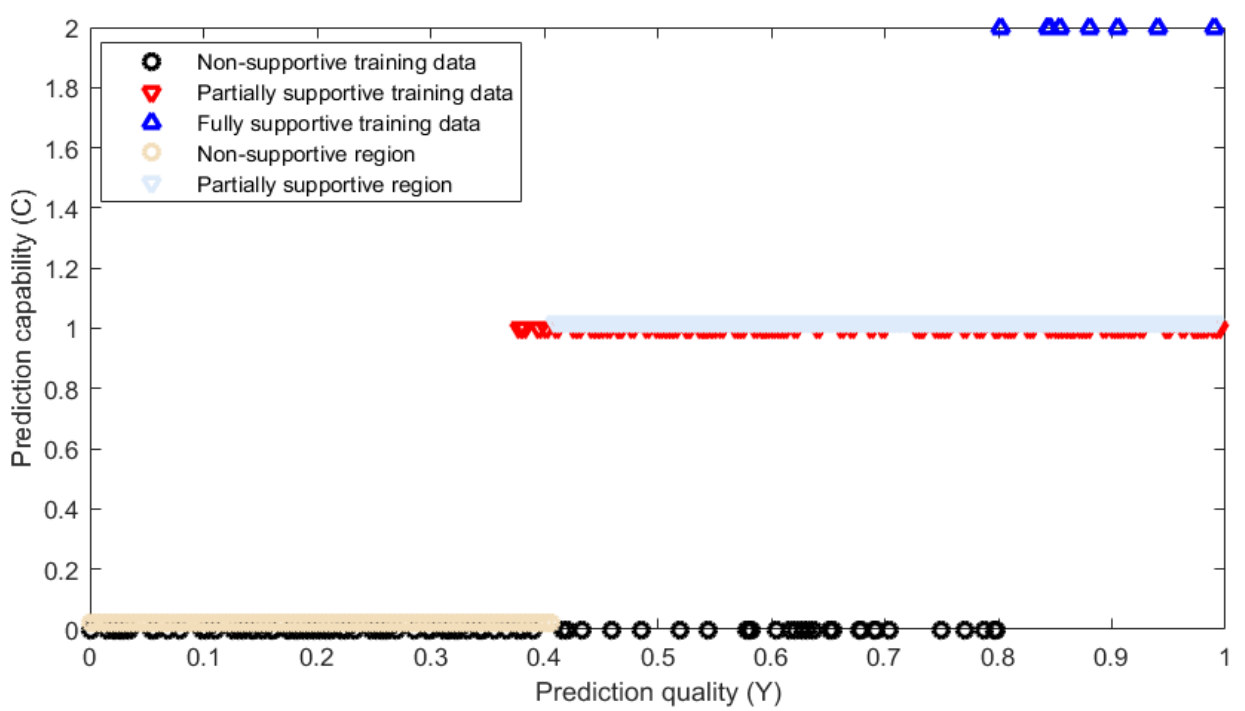

Figure 7 Training data and classifier when only $Y$ is considered

\section{3. Application}

In this section, the framework developed in Section 2 is implemented to assess the prediction capabilities

5 of three prognostic methods of literature, i.e., Fuzzy Similarity (FS) [38, 39], Feed-forward Artificial Neural

6 Networks (FANN) [40] and Hidden Semi-Markov Model (HSMM) [41-43]. A simulation case study of nine run-to-failure trajectories is considered as data, as shown in Figure 8 [31]. These data represent the failure

8 trajectories that can be extracted based on simulated mono-dimensional signal (e.g., temperature, pressure, or

9 vibration signal) of a generic component. The three methods are applied to predict the RUL, and their

10 prediction quality and method trustworthiness are assessed in Subsections 3.1 and 3.2, respectively. The

11 prediction capabilities of the three methods are determined in Subsection 3.3 by combining the prediction

12 quality and method trustworthiness. 

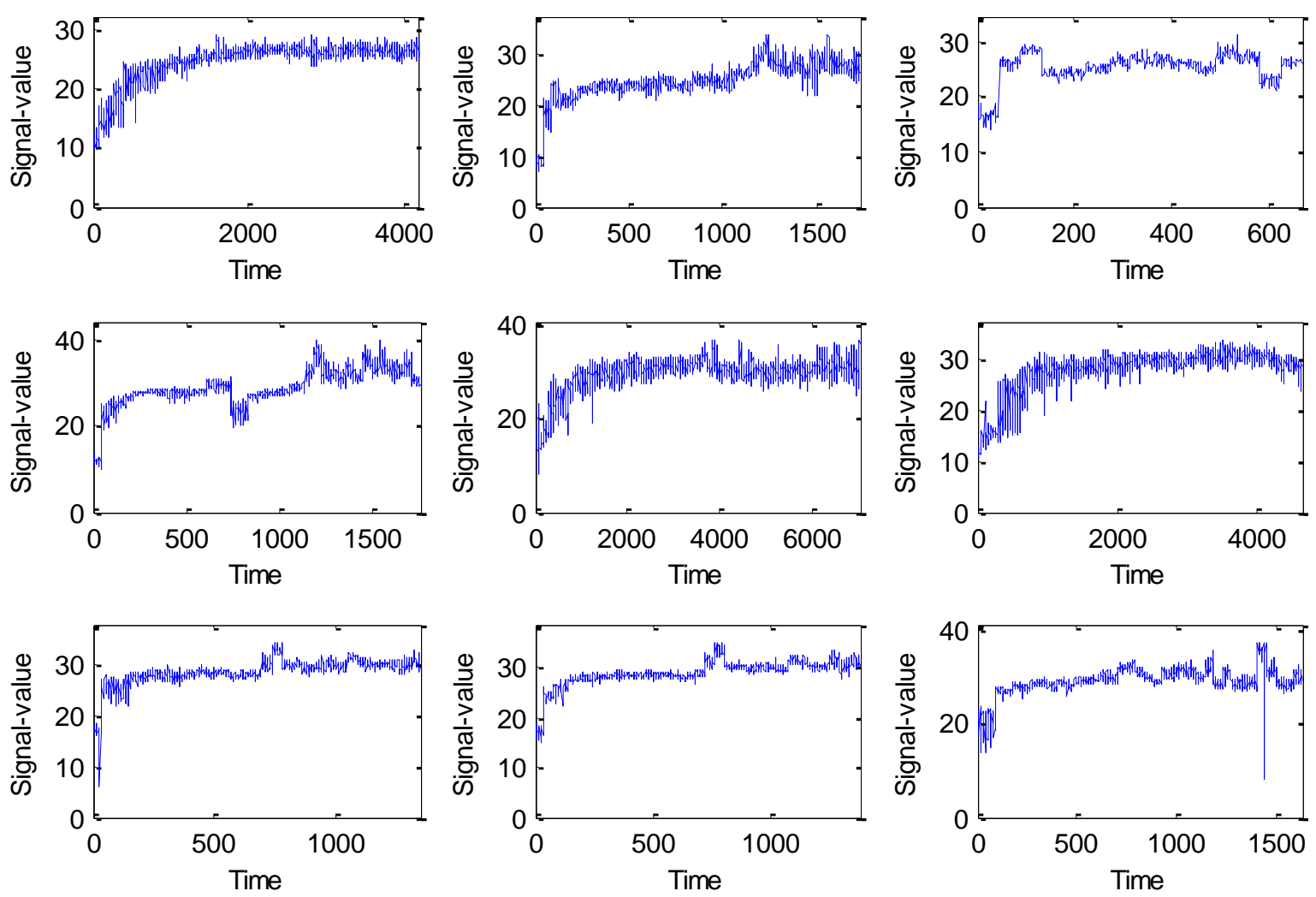

Figure 8 The nine simulated run-to-failure degradation trajectories

\subsection{Prediction quality assessment}

The three prognostic methods are applied to predict the RUL of the case study in Figure 8. Leave-one-out cross validations are used to compare the prediction quality of the three methods, where for each validation, one of the nine samples is left out while the rest eight are used as training samples. The RUL of the left-out sample is regarded as the true RUL so that the PPIs in Table A.1 can be calculated. Empirical Mode Decomposition (see [44] for details) is used for the three methods to pre-process the raw signal and construct

health indicators (HIs). The RUL prediction from each method is given in Figure 9. The accuracy and precision PPIs calculated based on Table A.1 are listed in Table 2 and Table 3. 

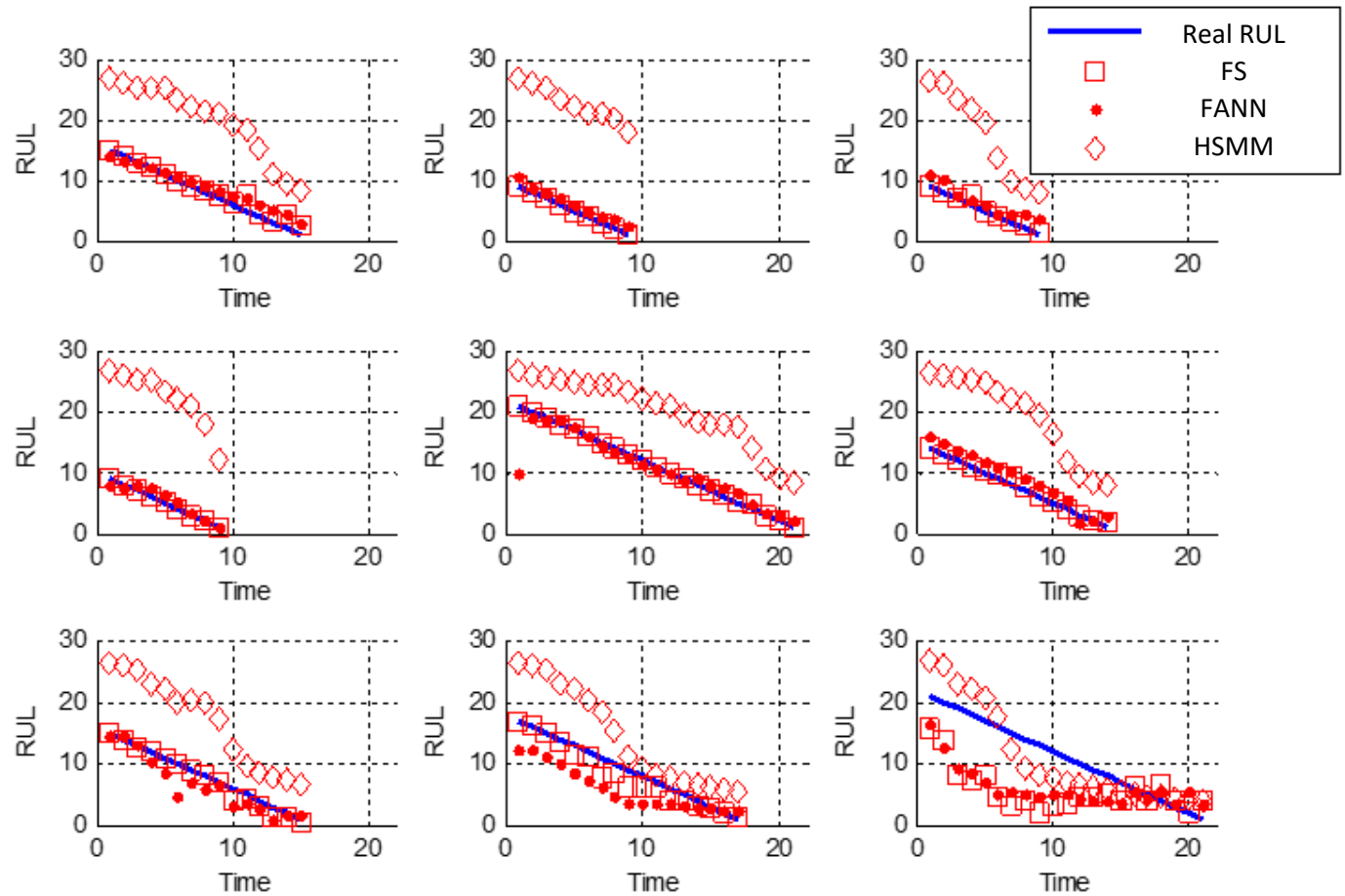

Figure 9 The predicted RUL of FS, FANN and HSMM [31]

Table 2 Accuracy PPIs for FS, FANN and HSMM

\begin{tabular}{|c|c|c|c|}
\hline PPIS & FS & FANN & HSMM \\
\hline$Y_{11}$ & 0.98 & 0.94 & 0.11 \\
\hline$Y_{12}$ & 0.37 & 0.56 & -9.47 \\
\hline$Y_{13}$ & 0.85 & 0.63 & -1.44 \\
\hline$Y_{14}$ & -5.14 & -7.56 & -143.79 \\
\hline$Y_{15}$ & 0.98 & 0.57 & -10.65 \\
\hline
\end{tabular}

Table 3 Precision PPIs for FS, FANN and HSMM

\begin{tabular}{|c|c|c|c|}
\hline PPIS & FS & FANN & HSMM \\
\hline$Y_{21}$ & 0.61 & 0.34 & 0.02 \\
\hline$Y_{22}$ & 0.97 & 0.94 & 0.67 \\
\hline$Y_{23}$ & -0.71 & -1.11 & -4.16 \\
\hline$Y_{24}$ & -0.28 & -1.42 & -10.20 \\
\hline$Y_{25}$ & 0.98 & 0.97 & 0.14 \\
\hline
\end{tabular}

6 To assess the values of $Y_{1}$ and $Y_{2}$, the weights of each Layer-4 basic sub-criteria should be determined first.

7 In this case study, experts are invited to rank all the PPIs in terms of their relative importance in affecting the

8 corresponding prognostic performance. Then, the weight of each PPI can be calculated by: 


$$
\omega^{(i)}=\frac{1-\frac{i-1}{n}}{\sum_{i=1}^{n}\left(1-\frac{i-1}{n}\right)}=\frac{2(n-i+1)}{n(n+1)},
$$

where $i$ is the ranking of the PPI (in descending order of importance) and $n$ is the total number of the PPIs in the same category. Suppose that $Y_{11}, Y_{12}, \cdots, Y_{15}$ and $Y_{21}, Y_{22}, \cdots, Y_{25}$ are in descending order of importance, respectively. According to (9), their weights are calculated and listed in Table 4.

Table 4 Weights of the PPIs

\begin{tabular}{|c|c|c|c|c|c|c|c|c|c|c|}
\hline & \multicolumn{5}{|c|}{ Accuracy PPIs } & \multicolumn{5}{c|}{ Precision PPIs } \\
\hline PPIs & $Y_{11}$ & $Y_{12}$ & $Y_{13}$ & $Y_{14}$ & $Y_{15}$ & $Y_{21}$ & $Y_{22}$ & $Y_{23}$ & $Y_{24}$ & $Y_{25}$ \\
\hline Weights & 0.333 & 0.267 & 0.200 & 0.133 & 0.067 & 0.333 & 0.267 & 0.200 & 0.133 & 0.067 \\
\hline
\end{tabular}

Then, the values of $Y_{1}$ and $Y_{2}$ are calculated based on (1) and the results are given in Table 5.

Table 5 Evaluation results of $Y_{1}$ and $Y_{2}$

\begin{tabular}{|c|c|c|c|c|c|}
\hline \multicolumn{2}{|c|}{ RUL point estimate prediction quality } & \multicolumn{4}{|c|}{ Uncertainty quantification quality } \\
\hline$Y_{1, F S}$ & $Y_{1, F A N N}$ & $Y_{1, H S M M}$ & $Y_{2, F S}$ & $Y_{2, F A N N}$ & $Y_{2, H S M M}$ \\
\hline-0.025 & -0.381 & -22.659 & 0.348 & 0.017 & -1.997 \\
\hline
\end{tabular}

The prediction quality $Y$ is, then, calculated based on (2) and Table 5, where the RUL point estimate quality and uncertainty quantification quality are assumed to have equal weights, $\omega_{1}=\omega_{2}=0.5$. The results are tabulated in Table 6 . The results in Table 6 show that considering both the point estimate and uncertainty quantification quality, Fuzzy Similarity performs the best among the three prognostic methods in terms of prediction quality, whereas the prediction quality of Hidden-Semi Markov Model is the worst among the three methods.

Table 6 Prediction quality of the three prognostic methods

\begin{tabular}{|c|c|c|}
\hline$Y_{F S}$ & $Y_{F A N N}$ & $Y_{H S M M}$ \\
\hline 0.433 & 0.307 & $1.63 \times 10^{-6}$ \\
\hline
\end{tabular}

\subsection{Method trustworthiness assessment}

\subsubsection{Step 1: Determine the inter-level priorities}

For the quantitative basic sub-criteria $X_{11}-X_{32}$, the numerical values for the criteria are collected in Table 7, where $M_{1}, M_{2}$ and $M_{3}$ correspond to FS, FANN and HSMM, respectively. Based on (3), the local priorities are calculated and given in Table 8. It should be noted that the numerical values in Table 7 are simulated for illustrative purposes. In practice, these values should be collected based on actual data extracted 
from literature and engineering applications.

Table 7 Numerical values for the basic sub-criteria

\begin{tabular}{|c|c|c|c|c|c|c|}
\hline & $X_{11}$ & $X_{12}$ & $X_{21}$ & $X_{22}$ & $X_{31}$ & $X_{32}$ \\
\hline$M_{1}$ & 24 & 18 & 22 & 16 & 22 & 18 \\
\hline$M_{2}$ & 39 & 31 & 41 & 38 & 38 & 31 \\
\hline$M_{3}$ & 38 & 32 & 35 & 24 & 28 & 22 \\
\hline
\end{tabular}

Table 8 Inter-level priorities of the alternative solutions with respect to $X_{11}-X_{32}$

\begin{tabular}{|c|c|c|c|c|c|c|}
\hline & $X_{11}$ & $X_{12}$ & $X_{21}$ & $X_{22}$ & $X_{31}$ & $X_{32}$ \\
\hline$p_{M_{1}, X_{i j}}$ & 0.238 & 0.222 & 0.224 & 0.205 & 0.250 & 0.254 \\
\hline$p_{M_{2}, X_{i j}}$ & 0.386 & 0.383 & 0.418 & 0.487 & 0.432 & 0.437 \\
\hline$p_{M_{3}, X_{i j}}$ & 0.376 & 0.395 & 0.357 & 0.308 & 0.318 & 0.310 \\
\hline
\end{tabular}

4

For the qualitative sub-criteria $X_{41}-X_{43}$, the local priorities are obtained by constructing pairwise comparison matrices. Altogether, there are eight pairwise comparison matrices that need to be constructed: one for the criteria in Layer 2, four for the sub-criteria in Layer 3 and three for the alternative solutions in Layer 4. For simplicity and illustrative purposes, we assume that all the criteria and sub-criteria in Layer 2 and Layer 3 are indifferent, so that all the elements in these pairwise comparison matrices are 1 and $p_{X_{1}}=\cdots=p_{X_{4}}=p_{X_{11}}=\cdots=p_{X_{43}}=0.5$. For the methods in Layer 4, experts are invited to make pairwise comparisons among the three methods in terms of computational costs, numbers of hyper-parameters and historical data requirements, respectively. The pairwise comparison matrices are constructed following the 1-9 scaling system introduced in Section 2.3. The resulted pairwise comparison matrices are

$$
A_{X_{41}}=\left[\begin{array}{ccc}
1 & 4 & 2 \\
1 / 4 & 1 & 1 / 3 \\
1 / 2 & 3 & 1
\end{array}\right], A_{X_{42}}=\left[\begin{array}{ccc}
1 & 6 & 4 \\
1 / 6 & 1 & 1 / 3 \\
1 / 4 & 3 & 1
\end{array}\right], A_{X_{43}}=\left[\begin{array}{ccc}
1 & 1 / 3 & 1 / 2 \\
3 & 1 & 2 \\
2 & 1 / 2 & 1
\end{array}\right] .
$$

The inter-level priorities are calculated using (4) and listed in Table 9. The value of $C R$ for each comparison matrix is also calculated following the procedures in Figure 4 to check the consistency. It can be seen from Table 9 that all the three $C R$ are less than the threshold value 0.1: therefore, all the three comparison matrices are consistent. 
Table 9 Inter-level priorities of the alternative solutions with respect to $X_{41}-X_{43}$

\begin{tabular}{|c|c|c|c|}
\hline & $X_{41}$ & $X_{42}$ & $X_{43}$ \\
\hline$p_{M_{1}, X_{i j}}$ & 0.558 & 0.691 & 0.163 \\
\hline$p_{M_{2}, X_{i j}}$ & 0.122 & 0.091 & 0.540 \\
\hline$p_{M_{3}, X_{i j}}$ & 0.320 & 0.218 & 0.297 \\
\hline$C R$ & 0.009 & 0.027 & 0.005 \\
\hline
\end{tabular}

2

\subsubsection{Step 2: Calculate the global priority for each alternative solution}

Equation (7) is, then, used to determine the global priority for each alternative solution, where the local priorities involved have been determined in Section 3.2.1 (see Table 8 and Table 9). The obtained global priorities are given in Table 10.

Table 10 Global priorities for the three prognostic methods

\begin{tabular}{|c|c|c|}
\hline$p_{M_{1}}$ & $p_{M_{2}}$ & $p_{M_{3}}$ \\
\hline 0.312 & 0.366 & 0.322 \\
\hline
\end{tabular}

\subsubsection{Step 3: Determine the method trustworthiness}

It can be seen from Table 10 that FANN $\left(M_{2}\right)$ is the most trustworthy one among the three prognostic methods. Its method trustworthiness is, then, evaluated by expert judgements and serves as benchmark for the other two methods. Suppose the experts judge that the trustworthiness of $M_{2}$ is $X_{M_{2}}=0.85$; then, the trustworthiness of the other two methods can be determined using (8), as shown in Table 11.

Table 11 Method trustworthiness for the three prognostic methods

\begin{tabular}{|c|c|c|}
\hline$X_{M_{1}}$ & $X_{M_{2}}$ & $X_{M_{3}}$ \\
\hline 0.72 & 0.85 & 0.75 \\
\hline
\end{tabular}

\subsection{Prediction capability assessment and method selection}

The prediction capabilities of the three prognostic methods are assessed using the classifier in Figure $\mathbf{6}$, where the values of $X$ and $Y$ are given in Table 11 and Table 6, respectively. The result is given in Table 12. Based on the assessment results, FS can be used to support CBM decision making for this specific application, while FANN and HSMM should not be used to support maintenance decisions due to their relative poor prediction capabilities in this case study. 


\begin{tabular}{|c|c|c|c|}
\hline Prognostic methods & FS $\left(M_{1}\right)$ & FANN $\left(M_{2}\right)$ & $\operatorname{HSMM}\left(M_{3}\right)$ \\
\hline$C_{M_{i}}$ & $C_{1}$ & $C_{0}$ & $C_{0}$ \\
\hline
\end{tabular}

\section{Conclusions}

In this paper, a hierarchical framework is developed to assess the prediction capability of prognostic methods. The framework considers the joint contributions from prediction quality and method trustworthiness (Layer 2). The prediction quality and method trustworthiness are further decomposed into six sub criteria (Layer 3) and 19 basic sub-criteria (Layer 4), where information and data can be collected to support the prediction capability assessment. A bottom-up method is developed to determine the prediction capability based on the information and data collected in the Layer-4 basic sub-criteria, in which the AHP method is applied for the aggregation of qualitative sub-criteria. A classification-based method is developed for the assessment of prediction capability. Based on the assessed prediction capability, the appropriateness of the prognostic method for supporting maintenance decisions can be determined, i.e., labelling it as qualified to support predictive maintenance, qualified to support condition-based maintenance or not qualified to support any maintenance decision. considered, nor rigidly prescriptive in the methods used for their evaluation. In the end, the prediction capability assessment is framed as a process of classification: given all the available information and knowledge, classify the prognostic methods based on their prediction capabilities. Therefore, in the future research, classification algorithms, e.g., Naïve Bayes classifier, majority rule sorting, etc., will also be investigated to develop efficient prediction capability assessment methods. Furthermore, various uncertainties exist in the process of prediction capability assessment. For example, the number of evidence in Figure 1 is often estimated based on sampling approaches. Hence, uncertainty arises from sampling errors. Also, the qualitative basic sub-criteria are evaluated based on pairwise comparisons and, therefore, subjected to uncertainty resulting from incomplete knowledge. How to address the effect of uncertainty in prediction capability assessment deserves further investigations too.

\section{Acknowledgement}

This work has been performed within the initiative of the Center for Resilience and Safety of Critical 
1 Infrastructures (CRESCI, http://cresci.cn). The major part of this paper was conducted during Dr. Zhiguo

2 Zeng's visit to Politecnico di Milano. The authors would also like to thank the editor and the two anonymous

3 reviewers for their help in improving the quality of this paper. 
Appendix Detailed definitions of the basic sub-criteria

Table A.1 Descriptions of the Layer-4 basic sub-criteria related to prediction quality

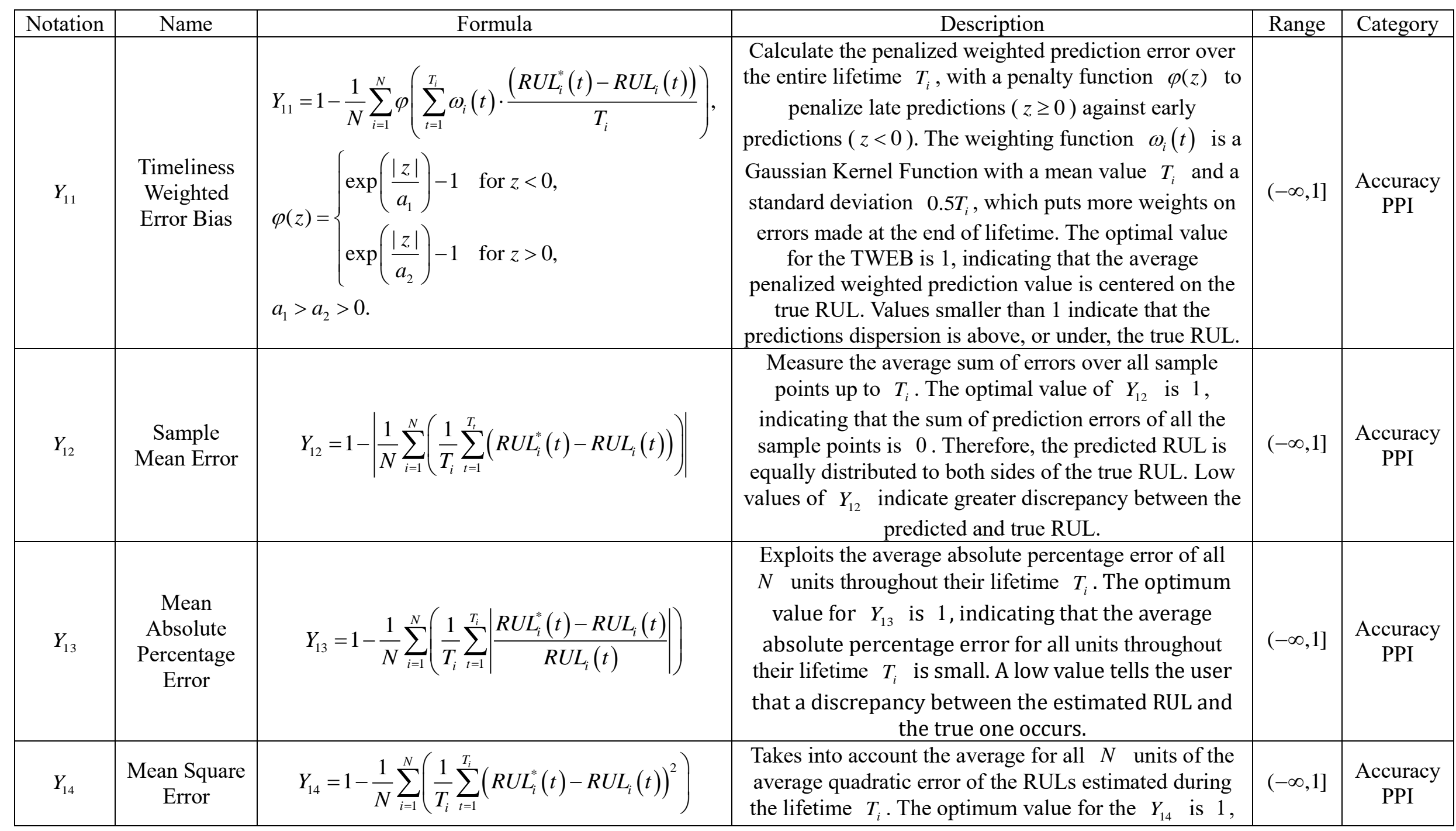




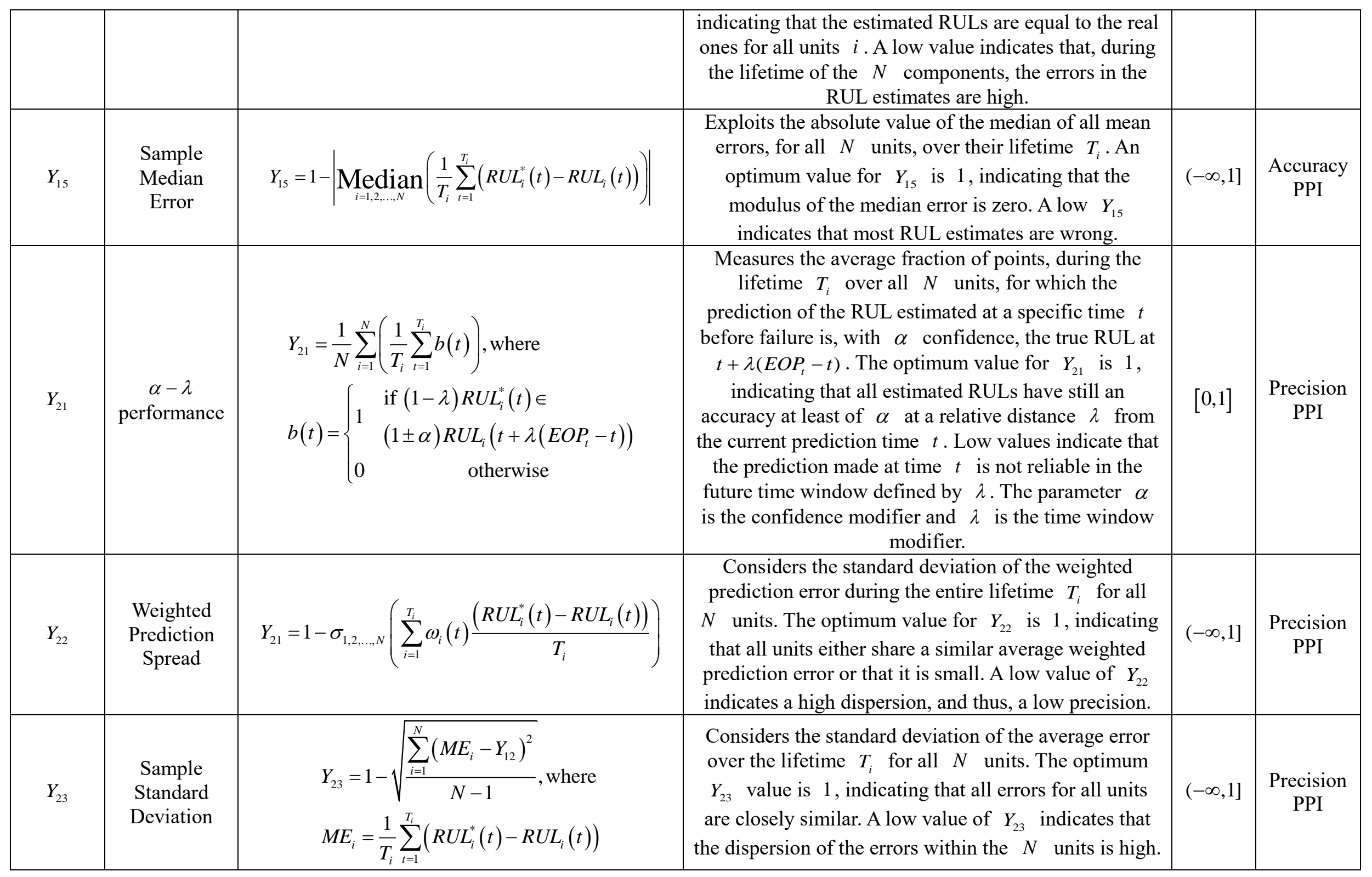




\begin{tabular}{|c|c|c|c|c|c|}
\hline$Y_{24}$ & $\begin{array}{l}\text { Root Mean } \\
\text { Square } \\
\text { Error }\end{array}$ & $Y_{24}=1-\frac{1}{N} \sum_{i=1}^{N} \sqrt{\frac{1}{T_{i}} \sum_{t=1}^{T_{i}}\left(R U L_{i}^{*}(t)-R U L_{i}(t)^{2}\right)}$ & $\begin{array}{l}\text { Considers the average of the Root Mean Squared Error } \\
\text { of the } N \text { units during the entire lifetime } T_{i} \text {. The } \\
\text { optimum value of } Y_{24} \text { is } 1 \text {, indicating that the error } \\
\text { between the estimated RUL and the true RUL is } \\
\text { consistent in the model. A low value indicates that the } \\
\text { discrepancy between the estimated and the true RUL is } \\
\text { inherently stochastic. }\end{array}$ & $(-\infty, 1]$ & $\begin{array}{l}\text { Precision } \\
\text { PPI }\end{array}$ \\
\hline$Y_{25}$ & $\begin{array}{l}\text { Prediction } \\
\text { Spread }\end{array}$ & $Y_{25}=1-\sigma_{1,2, \ldots, N}\left(M_{i}(t)\right)$ & $\begin{array}{l}\text { Considers the standard deviation of the Indicator } M \\
\text { for all } N \text { units. The } Y_{25} \text { measures how the indicator } \\
M \text { varies through all N units. The optimum value of } \\
Y_{25} \text { is } 1 \text {, indicating that the standard deviation of the } \\
\text { indicator is } 0 \text { : thus, the indicator is concentrated on one } \\
\text { value, reducing the variability of the performance } \\
\text { throughout the units. A low value indicates that the } \\
\text { indicators behavior varies between units. }\end{array}$ & $(-\infty, 1]$ & $\begin{array}{l}\text { Precision } \\
\text { PPI }\end{array}$ \\
\hline
\end{tabular}

1

2 Notations:

$3-i: \quad$ index for the identification of the unit under test (e.g., the equipment).

- $N:$ total number of units under test.

- $t:$ index for the time instant.

- $T$ : failure time of the unit. Note that each unit has a different $T_{i}$ value.

- EOP: End-Of-Prediction, time at which the unit is expected to fail, as predicted by the prognostic model.

- $n$ : number of total measurements.

- $R U L_{i}^{*}(t)$ : Estimated Remaining Useful Life (RUL) for the unit $i$, at time index $t$.

- $R U L_{i}(t)$ : Real RUL value for the unit $i$, at time index $t$.

- $M_{i}(t)$ : PPI calculated for the unit $i$, at time $t$. 
Table A.2 Descriptions of the Layer-4 basic sub-criteria related to method trustworthiness

\begin{tabular}{|c|c|c|c|}
\hline Notation & Meaning & Sub-criterion & Type \\
\hline$X_{11}$ & Number of academic evidence that supports the method's reliability & \multirow{2}{*}{ Reliability } & Quantitative \\
$X_{12}$ & Number of industrial evidence that supports the method's reliability & & Quantitative \\
\hline$X_{21}$ & Number of academic evidence that supports the method's validity & \multirow{2}{*}{ Validity } & Quantitative \\
$X_{22}$ & Number of industrial evidence that supports the method's validity & & Quantitative \\
\hline$X_{31}$ & Number of successful applications dealing with non-linear problems & \multirow{2}{*}{$\begin{array}{c}\text { Mathematical } \\
\text { modeling } \\
\text { adequacy }\end{array}$} & Quantitative \\
\hline$X_{32}$ & Number of successful applications dealing with non-stationary problems & Quantitative \\
\hline$X_{41}$ & Requirements on computational costs & \multirow{3}{*}{$\begin{array}{c}\text { Resources } \\
\text { requirements }\end{array}$} & Qualitative \\
\hline$X_{42}$ & Number of hyper-parameters that needs to be tuned & & Qualitative \\
\hline$X_{43}$ & Requirements on historical data & \\
\hline
\end{tabular}

2 
[1]. Pecht, M. and R. Jaai, A Prognostics and Health Management Roadmap for Information and Electronics-Rich Systems. Microelectronics Reliability, 2010. 50(3): p. 317-323.

[2]. Vichare, N.M. and M.G. Pecht, Prognostics and Health Management of Electronics. Components and Packaging Technologies, IEEE Transactions on, 2006. 29(1): p. 222-229.

[3]. Wang, H., Y. Li, Y. Liu, Y. Yang, and H. Huang, Remaining Useful Life Estimation Under Degradation and Shock Damage. Proceedings of the Institution of Mechanical Engineers Part O-Journal of Risk and Reliability, 2015. 229(3): p. 200-208.

[4]. Dalal, M., J. Ma and D. He, Lithium-Ion Battery Life Prognostic Health Management System Using Particle Filtering Framework. Proceedings of the Institution of Mechanical Engineers Part O-Journal of Risk and Reliability, 2011. 225(O1): p. 81-90.

[5]. Zhang, Z.X., X.S. Si, C.H. Hu, and X.Y. Kong, Degradation Modeling-Based Remaining Useful Life Estimation: A Review On Approaches for Systems with Heterogeneity. Proceedings of the Institution of Mechanical Engineers Part O-Journal of Risk and Reliability, 2015. 229(4SI): p. 343-355.

[6]. Tsui, K.L., N. Chen, Q. Zhou, Y.Z. Hai, and W.B. Wang, Prognostics and Health Management: A Review on Data Driven Approaches. Mathematical Problems in Engineering, 2015(793161).

[7]. Si, X.S., W.B. Wang, C.H. Hu, and D.H. Zhou, Remaining Useful Life Estimation - a Review On the Statistical Data Driven Approaches. European Journal of Operational Research, 2011. 213(1): p. 1-14.

[8]. Peng, Y., M. Dong and M.J. Zuo, Current Status of Machine Prognostics in Condition-Based Maintenance: A Review. The International Journal of Advanced Manufacturing Technology, 2010. 50(1-4): p. 297-313.

[9]. Lipi, T.F., J.H. Lim, M.J. Zuo, and W. Wang, A Condition-And Age-Based Replacement Model Using Delay Time Modelling. Proceedings of the Institution of Mechanical Engineers, Part O: Journal of Risk and Reliability, 2011: p. 1748006X11421265.

[10]. Yun, W.Y. and A.J. Endharta, A Preventive Replacement Policy Based On System Critical Condition. Proceedings of the Institution of Mechanical Engineers Part O-Journal of Risk and Reliability, 2016. 230(1SI): p. 93-100.

[11]. Saxena, A., S. Sankararaman and K. Goebel. Performance Evaluation for Fleet-Based and Unit-Based Prognostic Methods. in Second european conference of the prognostics and health management society. 2014

[12]. Saxena, A., J. Celaya, E. Balaban, K. Goebel, B. Saha, S. Saha, and M. Schwabacher. Metrics for Evaluating Performance of Prognostic Techniques. in Prognostics and health management, 2008. phm 2008. international conference on. 2008: IEEE.

[13]. Walther, B.A. and J.L. Moore, The Concepts of Bias, Precision and Accuracy, and their Use in Testing the Performance of Species Richness Estimators, with a Literature Review of Estimator Performance. Ecography, 2005. 28(6): p. 815-829.

[14]. Saxena, A., J. Celaya, B. Saha, S. Saha, and K. Goebel. On Applying the Prognostics Performance Metrics. in Annual Conference of the PHM Society. 2009.

[15]. Tobon-Mejia, D.A., K. Medjaher, N. Zerhouni, and G. Tripot, A Data-Driven Failure Prognostics Method Based On Mixture of Gaussians Hidden Markov Models. Reliability, IEEE Transactions on, 2012. 61(2): p. 491-503.

[16]. Micea, M.V., L. Ungurean, G.N. Cârstoiu, and V. Groza, Online State-Of-Health Assessment for Battery Management Systems. Instrumentation and Measurement, IEEE Transactions on, 2011. 60(6): p. 1997-2006.

[17]. Hu, Y., P. Baraldi, F. Di Maio, and E. Zio, Online Performance Assessment Method for a Model-Based Prognostic Approach. IEEE Transactions On Reliability, 2016. 2(65): p. 718-735.

[18]. Fan, J., K. Yung and M. Pecht, Prognostics of Lumen Maintenance for High Power White Light Emitting Diodes Using a Nonlinear Filter-Based Approach. Reliability Engineering \& System Safety, 2014. 123: p. 63-72.

[19]. Hu, Y., P. Baraldi, F. Di Maio, and E. Zio, A Particle Filtering and Kernel Smoothing-Based Approach for New Design Component Prognostics. Reliability Engineering \& System Safety, 2015. 134: p. 19-31.

[20]. Miao, Q., H. Huang and X. Fan, A Comparison Study of Support Vector Machines and Hidden Markov Models in Machinery Condition Monitoring. Journal of Mechanical Science and Technology, 2007. 21(4): p. 607-615.

[21]. Peng, T., Y. Liu, A. Saxena, and K. Goebel, In-Situ Fatigue Life Prognosis for Composite Laminates Based On Stiffness Degradation. Composite Structures, 2015. 132: p. 155-165.

[22]. Saxena, A., J. Celaya, B. Saha, S. Saha, and K. Goebel, Metrics for Offline Evaluation of Prognostic Performance. International Journal of Prognostics and Health Management, 2010. 1(1): p. 4-23.

[23]. Goebel, K., B. Saha and A. Saxena. A Comparison of Three Data-Driven Techniques for Prognostics. in 62nd meeting of the society for machinery failure prevention technology (mfpt). 2008.

[24]. Oberkampf, W.L., M. Pilch and T.G. Trucano, Predictive Capability Maturity Model for Computational Modeling and Simulation. 2007: Sandia National Laboratories.

[25]. Paulk, M.C., B. Curtis, M.B. Chrissis, and C.V. Weber, Capability Maturity Model, Version 1.1. Software, IEEE, 1993. 10(4): p. 18-27.

[26]. Gibson, C.F. and R.L. Nolan, Managing the Four Stages of EDP Growth. 1974: Harvard Business Review.

[27]. Herbsleb, J., D. Zubrow, D. Goldenson, W. Hayes, and M. Paulk, Software Quality and the Capability Maturity 
Model. Communications of the ACM, 1997. 40(6): p. 30-40.

[28]. Spruit, M. and K. Pietzka, MD3M: The Master Data Management Maturity Model. Computers in Human Behavior, 2015. 51(SIB): p. 1068-1076.

[29]. Farrell, M. and R. Gallagher, The Valuation Implications of Enterprise Risk Management Maturity. Journal of Risk and Insurance, 2015. 82(3): p. 625-657.

[30]. de Carvalho, J.V., A. Rocha and J. Vasconcelos, Towards an Encompassing Maturity Model for the Management of Hospital Information Systems. Journal of Medical Systems, 2015. 39(999).

[31]. Di Maio, F., P. Turati and E. Zio. Prediction Capability Assessment of Data-Driven Prognostic Methods for Railway Applications. in Third European conference of the prognostic and health management society. 2016. At Bilbao, Spain: PHM Society.

[32]. Saaty, T.L., A Scaling Method for Priorities in Hierarchical Structures. Journal of Mathematical Psychology, 1977. 15(3): p. 234-281.

[33]. Streimikiene, D., J. Sliogeriene and Z. Turskis, Multi-Criteria Analysis of Electricity Generation Technologies in Lithuania. Renewable Energy, 2016. 85: p. 148-156.

[34]. Vaidya, O.S. and S. Kumar, Analytic Hierarchy Process: An Overview of Applications. European Journal of Operational Research, 2006. 169(1): p. 1-29.

[35]. Huguenin, J., Data Envelopment Analysis and Non-Discretionary Inputs: How to Select the Most Suitable Model Using Multi-Criteria Decision Analysis. Expert Systems with Applications, 2015. 42(5): p. 2570-2581.

[36]. Dožić, S. and M. Kalić, An AHP Approach to Aircraft Selection Process. Transportation Research Procedia, 2014. 3: p. 165-174.

[37]. Saaty, T.L., Decision Making with the Analytic Hierarchy Process. International journal of services sciences, 2008. 1(1): p. 83-98.

[38]. Di Maio, F. and E. Zio, Failure Prognostics by a Data-Driven Similarity-Based Approach. International Journal of Reliability, Quality and Safety Engineering, 2013. 20(1).

[39].Zio, E. and F. Di Maio, A Data-Driven Fuzzy Approach for Predicting the Remaining Useful Life in Dynamic Failure Scenarios of a Nuclear System. Reliability Engineering and System Safety, 2010. 95(1): p. 49-57.

[40].Bai, G., P. Wang and C. Hu, A Self-Cognizant Dynamic System Approach for Prognostics and Health Management. Journal of Power Sources, 2014. 278: p. 163-174.

[41]. Dong, M. and D. He, A Segmental Hidden semi-Markov Model (HSMM)-based Diagnostics and Prognostics Framework and Methodology. Mechanical Systems and Signal Processing, 2007. 21(5): p. 2248-2266.

[42]. Moghaddass, R. and M.J. Zuo, An Integrated Framework for Online Diagnostic and Prognostic Health Monitoring Using a Multistate Deterioration Process. Reliability Engineering \& System Safety, 2014. 124: p. 92-104.

[43]. Miao, Q. and V. Makis, Condition Monitoring and Classification of Rotating Machinery Using Wavelets and Hidden Markov Models. Mechanical Systems and Signal Processing, 2007. 21(2): p. 840-855.

[44]. Huang, N.E., Z. Shen, S.R. Long, M.C. Wu, H.H. Shih, Q. Zheng,... H.H. Liu. The Empirical Mode Decomposition and the Hilbert Spectrum for Nonlinear and Non-Stationary Time Series Analysis. in Proceedings of the Royal Society of London A: Mathematical, Physical and Engineering Sciences. 1998: The Royal Society. 